\title{
Preoperative pain measurements in correlation to deep endometriosis classification with Enzian.
}

\author{
Jeroen Metzemaekers ${ }^{1}$, Mathijs Blikkendaal ${ }^{2}$, Kim Nieuwenhuizen ${ }^{1}$, Kim Bronsgeest ${ }^{1}$, \\ Johann Rhemrev ${ }^{2}$, Mathilde Smeets ${ }^{2}$, James English ${ }^{2}$, Frank Willem Jansen ${ }^{1}$, and Andries \\ Twijnstra $^{1}$ \\ ${ }^{1} \mathrm{LUMC}$ \\ ${ }^{2}$ Medical Centre Haaglanden
}

November 13, 2021

\begin{abstract}
Objective To study pain symptoms and their correlation with the anatomical location (and extent) of deep endometriosis lesion(s) classified by the Enzian score. Design Prospective multi-centre study Setting Web-based application called EQUSUM (www.equsum.org) to classify and report surgical procedures Population or Sample A total of 419 surgical DE (deep endometriosis) cases Methods Collection of surgical data in DE cases and their endometriosis classification and pain scores. Main Outcome Measures Preoperative reported pain scores in each domain (dysmenorrhea, dyschezia, dysuria, dyspareunia, chronic pelvic pain) were collected along with the Enzian classification. Baseline characteristics, pain scores, surgical procedure and extent of the disease were also collected. Results In general, more extensive involvement of DE does not lead to an increase in numerical rating scale for pain measures. However, dysuria and bladder involvement do show a clear correlation AUC 0.62 (SE 0.04, CI 0.54-0.71, $\mathrm{p}<0.01$ ). Regarding the predictive value of dyschezia, we found a weak, but significant correlation with ureteric involvement; AUC 0.60 (SE 0.04, CI 0.53-0.67, p< 0.01). Conclusions Pain symptoms poorly correlate with anatomical locations of deep endometriosis in almost all pain scores, with the exception of bladder involvement and dysuria which did show a correlation. Also dyschezia seems to have predictive value for DE ureteric involvement and therefore MRI or ultrasound imaging (ureter and kidney) is recommended in the preoperative workup in these patients.
\end{abstract}

Preoperative pain measurements in correlation to deep endometriosis classification with Enzian.

Running title: Correlation of pain and deep endometriosis

J. Metzemaekers ${ }^{\mathrm{a}}$, M.D. Blikkendaal ${ }^{\mathrm{b}}$, K.E. v. Nieuwenhuizen ${ }^{\mathrm{a}}$, K. Bronsgeest ${ }^{\mathrm{a}}$, J.P.T. Rhemrev ${ }^{\mathrm{b}}$, M.J.G.H. Smeets $^{\mathrm{b}}$, J. English ${ }^{\mathrm{b}}$, F.W. Jansen ${ }^{\mathrm{a}, \mathrm{c}}$, A.R.H.Twijnstra ${ }^{\mathrm{a}}{ }^{*}$

${ }^{a}$ Department of Gynaecology, Leiden University Medical Center, Leiden, the Netherlands.

b Department of Gynaecology, Haaglanden Medisch Centrum-Bronovo, Den Haag, the Netherlands.

${ }^{c}$ Department of Biomechanical Engineering, Delft University of Technology, Delft, the Netherlands.

* Corresponding author contact information:

dr. A.R.H. Twijnstra, MD, PhD Department of Gynaecology Leiden University Medical Center

PO Box 9600, 2300 RC Leiden, the Netherlands E-mail: a.r.h.twijnstra@lumc.nl

\section{Abstract}

Objective 
To study pain symptoms and their correlation with the anatomical location (and extent) of deep endometriosis lesion(s) classified by the Enzian score.

\section{Design}

Prospective multi-centre study

\section{Setting}

Web-based application called EQUSUM (www.equsum.org) to classify and report surgical procedures

\section{Population or Sample}

A total of 419 surgical DE (deep endometriosis) cases

\section{Methods}

Collection of surgical data in DE cases and their endometriosis classification and pain scores.

\section{Main Outcome Measures}

Preoperative reported pain scores in each domain (dysmenorrhea, dyschezia, dysuria, dyspareunia, chronic pelvic pain) were collected along with the Enzian classification. Baseline characteristics, pain scores, surgical procedure and extent of the disease were also collected.

\section{Results}

In general, more extensive involvement of $\mathrm{DE}$ does not lead to an increase in numerical rating scale for pain measures. However, dysuria and bladder involvement do show a clear correlation AUC 0.62 (SE 0.04, CI 0.54-0.71, $\mathrm{p}<0.01)$. Regarding the predictive value of dyschezia, we found a weak, but significant correlation with ureteric involvement; AUC 0.60 (SE 0.04, CI 0.53-0.67, p< 0.01).

\section{Conclusions}

Pain symptoms poorly correlate with anatomical locations of deep endometriosis in almost all pain scores, with the exception of bladder involvement and dysuria which did show a correlation. Also dyschezia seems to have predictive value for DE ureteric involvement and therefore MRI or ultrasound imaging (ureter and kidney) is recommended in the preoperative workup in these patients.

\section{Funding:}

The author(s) received no financial support for the research, authorship, and/or publication of this article.

\section{Key words:}

Endometriosis, surgery, rASRM, Enzian, classification

\section{Tweetable abstract:}

Poor correlation of pain intensity and anatomical locations of deep endometriosis nodules in patients with deep endometriosis.

\section{Introduction}

An ideal classification system should meet three core principles: it should be simple, allow description of the disease and correlate with clinical findings (1). Currently, the two most used classifications systems for endometriosis are the rASRM (2) and Enzian (3), of which neither shows strong clinical correlation with pain symptoms $(4,5)$. This could be explained by the fact that the rASRM is not directly suitable to describe deep endometriosis (6). On the other hand, the Enzian classification is not able to describe the tubes, ovaries and peritoneum which is covered by the rASRM classification. An attempt to overcome these difficulties has recently been made by theStiftung Endometriose Forschung (SEF) at their annual Weisensee endometriosis meeting, during which an update of the Enzian classification was created: the \#Enzian (7). 


\section{Clinical correlations with the Enzian}

Previous studies (4) (8) reported the correlations between the Enzian and pain symptoms in deep endometriosis. Haas et al reported no combinations of compartments, but choose to report the compartment with the highest severity: for example, A1B0C3 would become a solitary $\mathrm{C}$ in the analysis. In real life, endometriosis does not respect the compartment boundaries of the Enzian classification and therefore it is difficult to translate these findings directly to the clinical symptoms. Montanari et al performed a similar analysis with a more robust cohort. Nevertheless, both studies failed to study the solitary compartments and combinations of compartments in correlation to pain symptoms.

Therefore, our research questions that we addressed are: 1) Which compartment is responsible for the severity of the different types of pain? 2) Does pain increase with an increase in compartment involvement. 3) Do specific clinical symptoms correlate with the scoring in the Enzian.

The primary aim of our study was to find a correlation of the Enzian score with pain symptoms in DE. Secondly, we wished to determine whether a correlation exists between the extent of the disease and the reported intensity of pain (regarding solitary compartments and combinations of compartments).

\section{Methods}

\section{Study design}

We performed a multi-centre study (university hospitals, non-university hospitals and specialised clinics for endometriosis) with prospectively collected surgical data in DE cases and their endometriosis classification from 6 European countries.

\section{Data collection}

Data was collected from February 2019 through June 2020 with the EQUSUM application (9). Registered data included: general data on patients' characteristics, previous abdominal surgery, pain scores for dysmenorrhoea, dyschezia, dysuria, chronic pelvic pain, dyspareunia, accurate localisation of endometriosis lesions, and in case of fertility wish, the EFI scores (9). The EQUSUM application automatically generates the following classifications: rASRM, Enzian and EFI scores. For this research question only the Enzian classification is studied.

The inclusion criterion was the presence of deep endometriosis in one or more of the main Enzian compartments A, B, C or F. Deep endometriosis was staged on the clinical intra-operative view combined with preoperative imaging.

Exclusion criteria were diagnostic laparoscopy procedures or procedures without disease involvement in one of the main compartments (A,B,C or F). Pain scores were preoperatively documented with a numeric rating scale (NRS) ranging from 0-10. The Enzian scores were assigned according to the original manuscript (3).

\section{DE and not DIE}

For this study we did not use the definition of Koninckx et al., in 1990 for deep infiltrating endometriosis (DIE) (10), which can only be confirmed by the pathologist (endometrial implants penetrate more than $5 \mathrm{~mm}$ into the peritoneum). We used the definition described by Donnez (11), which is a nodular form of deep endometriosis (DE) which we classified with the Enzian based on clinical expertise combined with radiological and intra-operative findings.

\section{Compartment involvement}

Main pain scores were calculated for all main compartments solitary. This implies that for example only options of $\mathrm{A} 1 / 2 / 3, \mathrm{~B} 0, \mathrm{C} 0, \mathrm{Fx}$ would be assigned to compartment A. Combinations were also possible, for example $\mathrm{A} 1 \mathrm{~B} 2 \mathrm{C} 0$, would result in the group $\mathrm{AB}$ and so on. The assignment to the main compartments was irrespective of the involvement of F. Solitary mean pain calculations for the compartments F (FB, FA, 
FU and FI). This was performed to present the pain scores with the least bias of other compartments. To present the overlay of certain compartments we used euler diagrams (12).

Predictive value of pain symptoms in correlation to endometriosis involvement with the Receiver Operating Characteristic curve

To test if endometriosis pain symptoms could predict deep endometriosis involvement, we calculated receiver operating characteristic (ROC) curves. The test in this case is the presence of endometriosis classified with the Enzian (involvement yes or no), and the continuous variable is the severity of symptoms measured with the NRS scale (0-10).

\section{Data analysis}

IBM SPSS version 25.0 for Windows was used for our analysis and we used the Shapiro-Wilk test to evaluate the distribution of the data. Data are presented as mean \pm standard deviation (SD) or median (with interquartile range) for normally distributed or skewed data, respectively. Euler eclips diagrams were created with online software based on an R package called eulerr (12). To test the accuracy of symptoms in correlation to the anatomical location, ROC curves and the areas under the curves were obtained for each anatomical location. We considered a 2 -tailed p-value of $<0.05$ as statistically significant. And for the ROC curve we considered an area under the curve of [?]0.6 as a discriminating test (13).

\section{Results}

\section{Study population}

In total 475 procedures were registered. Nine cases were removed because of diagnostic procedures and 47 cases were removed because no deep endometriosis was present in compartment A, B, C or F. This resulted in a total of $419 \mathrm{DE}$ cases. Baseline characteristics are presented in Table 1. The mean age of women who underwent surgery was $35.3+-6.5$ years, with a median BMI of 24 (IQR 21-28) kg/m². The majority of women had one or more previous abdominal surgeries; $13 \%$ reported more than 2 procedures. The primary indication for surgery were pain symptoms $(82 \%)$.

Table 2 shows all classification types. Regarding the Enzian classification, compartment A was involved in 110 cases $(26.3 \%)$, with a majority being A3 (55.5\%). Compartment B was reported in 244 cases $(58.2 \%)$ on the left side and 229 cases (52.3\%) on the right side. The majority had a B2 severity $(50.4 \%$ left and $53.7 \%$ right). Sacro-uterine involvement was reported the most, and cardinal ligaments the least. Compartment $\mathrm{C}$ was reported in 189 cases (45.1\%), with the majority having a C3 severity (55.6\%). Adenomyosis (FA) was reported in $58 \%$ of all cases, with a majority of the diffuse type (62.6\%). Bladder (FB) involvement was reported in 71 cases (16.9\%). Intestinal involvement (FI) was reported in 94 cases (22.4\%), with $49.6 \%$ rectum involvement cranial to the recto-sigmoid junction, appendix involvement in $33.6 \%$, ileocecal in $7.6 \%$, coecum in $6.7 \%$ and ileum in $2.5 \%$ of the FI cases. Ureteric involvement (FU left) was reported in 60 cases $(14.3 \%)$, with hydronephrosis in 6 cases (10\%). FU on the right side was reported in 44 cases (10.5\%), with 6 cases hydronephrosis (13.6\%). Other Enzian involvement (FO) was reported in 23 cases (5.5\%), with $95.6 \%$ umbilical and $4.3 \%$ diaphragmatic lesions.

\section{Preoperative numeric pain scale}

Table 3 shows the median pain scores, ranging from most painful (dysmenorrhea) to least painful (dysuria). For dysmenorrhea (NRS 0-10) a median of 8 (IQR 7-9) was reported. A mean dyschezia of 4.9+-3.7, dyspareunia 4.5+-3.5, chronic pelvic pain (CPP) 4.5+-3.5 and dysuria symptoms with a mean pain score of 2.1+-3.0.

Solitary and grouped pain scores (0-10) for the different Enzian compartments are presented in Figure 1.

Dysmenorrhoea figure 1.1 (red) 
A mean pain for compartment A was found of 5.5, SE $2.10(\mathrm{n}=6)$, B 7.4, SE $0.27(\mathrm{n}=125)$, C 7.3, SE 0.54 $(\mathrm{n}=34)$, A\&B 7.5 SE $0.62(\mathrm{n}=24)$, A\&C 6.5 SE $0.68(\mathrm{n}=19)$, B\&C 7.6 SE 0.33(n=75) and A\&B\&C 7.3 SE $0.39(n=61)$. For the F compartments: FB 5.6 SE $1.42(n=10)$, FA 6.8 SE $0.47(n=63)$, FI 5.1 SE 1.15 $(\mathrm{n}=15)$, FU 5.4 SE $2.27(\mathrm{n}=6)$. No dysmenorrhoea but with Enzian involvement is present in Supplementary table $\mathrm{S} 1 \mathrm{n}=1(16.7 \%)$ in compartment $\mathrm{A}, \mathrm{n}=10(8 \%)$ in compartment $\mathrm{B}, \mathrm{n}=3(8.8 \%)$ in compartment $\mathrm{C}$, $\mathrm{n}=3(30 \%)$ in compartment $\mathrm{FB}, \mathrm{n}=11(17.5 \%)$ in compartment $\mathrm{FA}, \mathrm{n}=4(26.7 \%)$ in compartment $\mathrm{FI}$ and $\mathrm{n}=2(33.3 \%)$ in $\mathrm{FU}$.

Dyschezia figure 1.2 (purple)

A mean pain for compartment A was found of 5.0, SE $2.05(\mathrm{n}=6)$, B 4.5, SE $0.36(\mathrm{n}=125)$, C 5.4, SE 0.63 $(\mathrm{n}=34)$, A\&B 6.2 SE $0.73(\mathrm{n}=24)$, A\&C 6.1 SE 0.76 $(\mathrm{n}=19)$, B\&C 5.6 SE 0.41 $(\mathrm{n}=75)$ and A\&B\&C 5.8 SE $0.43(\mathrm{n}=61)$. For the F compartments: FB 2.4 SE 1.25 (n=10), FA 3.0 SE $0.46(\mathrm{n}=63)$, FI 4.7 SE 1.12 $(\mathrm{n}=15)$, FU 4.2 SE $1.90(\mathrm{n}=6)$. No dyschezia but with Enzian involvement is present in Supplementary table $\mathrm{S} 1 \mathrm{n}=2(33.3 \%)$ in compartment $\mathrm{A}, \mathrm{n}=40(32.0 \%)$ in compartment $\mathrm{B}, \mathrm{n}=8(23.5 \%)$ in compartment $\mathrm{C}, \mathrm{n}=7$ $(70.0 \%)$ in compartment $\mathrm{FB}, \mathrm{n}=31(49.2 \%)$ in compartment $\mathrm{FA}, \mathrm{n}=5(33.3 \%)$ in compartment $\mathrm{FI}$ and $\mathrm{n}=3$ $(50.0 \%)$ in FU.

Chronic pelvic pain figure 1.3 (grey)

A mean pain for compartment A was found of 5.0, SE $1.10(\mathrm{n}=6)$, B 4.6, SE $0.33(\mathrm{n}=125)$, C 4.5, SE 0.59 $(\mathrm{n}=34), \mathrm{A} \& \mathrm{~B} 4.4 \mathrm{SE} 0.79(\mathrm{n}=24)$, A\&C 3.5 SE $0.81(\mathrm{n}=19), \mathrm{B} \& \mathrm{C} 4.8$ SE $0.39(\mathrm{n}=75)$ and A\&B\&C 4.1 SE $0.45(\mathrm{n}=61)$. For the F compartments: FB 1.7 SE $1.14(\mathrm{n}=10)$, FA 5.0 SE $0.47(\mathrm{n}=63)$, FI 4.5 SE $1.18(\mathrm{n}=15), \mathrm{FU} 7.33 \mathrm{SE} 1.54(\mathrm{n}=6)$. No chronic pelvic pain but with Enzian involvement is present in Supplementary table S1 $\mathrm{n}=0(0.0 \%)$ in compartment A, $\mathrm{n}=38(30.4 \%)$ in compartment B, $\mathrm{n}=10(29.4 \%)$ in compartment $\mathrm{C}, \mathrm{n}=8(80.0 \%)$ in compartment $\mathrm{FB}, \mathrm{n}=18(28.6 \%)$ in compartment $\mathrm{FA}, \mathrm{n}=6(40.0 \%)$ in compartment FI and $\mathrm{n}=1(16.7 \%)$ in FU.

Dyspareunia figure 1.4 (blue)

A mean pain for compartment A was found of 3.8, SE $1.59(\mathrm{n}=6)$, B 4.5, SE $0.35(\mathrm{n}=125)$, C 3.9, SE 0.58 $(\mathrm{n}=34)$, A\&B 3.8 SE $0.70(\mathrm{n}=24)$, A\&C 3.7 SE $0.82(\mathrm{n}=19)$, B\&C 4.4 SE $0.43(\mathrm{n}=75)$ and A\&B\&C 4.6 SE $0.44(\mathrm{n}=61)$. For the F compartments: FB 4.6 SE $1.09(\mathrm{n}=10)$, FA 5.7 SE $0.41(\mathrm{n}=63)$, FI 4.1 SE 1.02 $(\mathrm{n}=15)$, FU 6.0 SE 1.29 $(\mathrm{n}=6)$. No dyspareunia but with Enzian involvement is present in Supplementary table S1 $\mathrm{n}=2(33.3 \%)$ in compartment $\mathrm{A}, \mathrm{n}=41(32.8 \%)$ in compartment $\mathrm{B}, \mathrm{n}=12(35.3 \%)$ in compartment $\mathrm{C}, \mathrm{n}=3(30.0 \%)$ in compartment $\mathrm{FB}, \mathrm{n}=11(17.5 \%)$ in compartment $\mathrm{FA}, \mathrm{n}=6(40.0 \%)$ in compartment $\mathrm{FI}$ and $\mathrm{n}=1(16.7 \%)$ in $\mathrm{FU}$.

Dysuria figure 1.5 (yellow)

A mean pain for compartment A was found of 1.4, SE 1.4 (n=6), B 1.7, SE 0.25 (n=125), C 1.9, SE 0.51 $(\mathrm{n}=34)$, A\&B 2.6 SE $0.68(\mathrm{n}=24)$, A\&C 1.5 SE 0.55 $(\mathrm{n}=19)$, B\&C 1.7 SE 0.34(n=75) and A\&B\&C 2.3 SE $0.40(\mathrm{n}=61)$. For the F compartments: FB 7.1 SE $0.89(\mathrm{n}=10)$, FA 2.3 SE $0.42(\mathrm{n}=63)$, FI 2.43 SE 1.00 $(\mathrm{n}=15)$, FU 4.2 SE $1.60(\mathrm{n}=6)$. No dysuria but with Enzian involvement is present in Supplementary table $\mathrm{S} 1 \mathrm{n}=4(66.7 \%)$ in compartment $\mathrm{A}, \mathrm{n}=73(58.4 \%)$ in compartment $\mathrm{B}, \mathrm{n}=22(64.7 \%)$ in compartment $\mathrm{C}, \mathrm{n}=1$ $(10.0 \%)$ in compartment $\mathrm{FB}, \mathrm{n}=35(55.6 \%)$ in compartment FA, $\mathrm{n}=9(60.0 \%)$ in compartment $\mathrm{FI}$ and $\mathrm{n}=2$ $(33.3 \%)$ in $\mathrm{FU}$.

\section{ROC curve for ureter and bladder}

ROC curves in correlation to the median pain scores were calculated for the Enzian locations A, B, C, FB, FU, FA and FI. We only present the ROC curves with an area under the curve [?]0.6, since in that case this test can provide a discrimination. This was only the case for the ureter and bladder involvement (table 4).

Figure 2.1 shows the ROC curve for the ureter, with an area under the curve for dysmenorrhea of 0.52 (SE 0.04, CI 0.45-0.60, p=0.04), dysuria 0.51 (SE 0.04, CI 0.44-0.59, p=0.68), CPP 0.52 (SE 0.04, CI 0.44-0.60, 
$\mathrm{p}=0.67$ ), dyspareunia 0.49 (SE 0.04, CI 0.41-0.56, $\mathrm{p}=0.70$ ) and dyschezia 0.6 (SE 0.04, CI 0.53-0.67, $\mathrm{p}<$ 0.01) (table 4). We chose the cut-off point for dyschezia of NRS 6.50, while a high sensitivity is important regarding ureter involvement (cave silent hydronephrosis). Using this as a cutoff point, our sensitivity (true positive rate) would be $63 \%$ and our 1 - specificity would be $45 \%$ (false positive rate).

For the bladder (figure 2.2), with an area under the curve for dysmenorrhea of 0.49 (SE 0.04, CI 0.41-0.56, $\mathrm{p}=0.73$ ), dysuria 0.62 (SE 0.04, CI 0.54-0.71, p $<0.01$ ), CPP 0.39 (SE 0.04, CI 0.31-0.47, $\mathrm{p}<0.01$ ), dyspareunia 0.44 (SE 0.04, CI 0.36-0.52, $\mathrm{p}=0.13$ ) and dyschezia 0.57 (SE 0.04, CI 0.49-0.66, $\mathrm{p}<0.07$ ) (table 4). We chose the cut-off point for dysuria of NRS 0.5. Using this as a cutoff point, our sensitivity would be $54 \%$ and our 1 - specificity would be $36 \%$.

\section{Discussion}

\section{Main findings}

Preoperative reported endometriosis related pain scores correlate poorly with intra-operative of the locations of DE lesions. This underlines and explains the difficulty doctors and patients face, before the diagnosis endometriosis is set (an average of 9 years) (14). The correlation of endometriosis involvement to pain symptoms is not a 1-on-1 relationship, this partly explains why undiagnosed patients often see numerous doctors and try several treatment options before the diagnosis endometriosis is made. This delay makes women lose faith in the healthcare system or they start doubting themselves (Are my symptoms real? Nobody is able to find anything) (15). When the diagnosis is finally made, surgery for removing the endometriosis is not the only solution. These women often developed chronic pain symptoms which need a broader treatment process than solely removing the physical stimuli (endometriosis nodules). A more holistic approach, which also includes psychological support and pain management/insight is mandatory to help these women regain their normal life (16).

\section{Interpretation}

\section{Pain is more than a physical stimulus from an anatomical location}

The theory that endometriosis has a multifactorial pathogenesis could explain why endometriosis remains difficult to classify regarding prognosis and clinical correlation. This multifactorial pathogenesis includes endometriosis severity (stage, location, depth of invasion), co-morbidity conditions, myofascial factors (muscle and fascial involvement), and central sensitisation (17). According to the International Association for the Study of Pain, the definition of pain is "an unpleasant sensory and emotional experience associated with actual or potential tissue damage". Besides, it has become more clear that pain experience is also influenced by psychological factors (e.g. catastrophising, anxiety, coping) $(18,19)$ and central sensitisation (persistent state of high reactivity) (20). This supports the theory that not only the severity of the endometriosis is responsible for pain symptoms. Endometriosis lesions on anatomical structures are not solely responsible for the pain sensation: it seems far more complex than that.

\section{No pain symptoms, but DE is present}

It is interesting to find that one third of the patients do not report dyschezia, but do have intestinal DE involvement. This phenomenon is present in more compartments like adenomyosis whereby $17 \%$ do not report dysmenorrhea and $24 \%$ of the cases do not report dyschezia, but there is rectal involvement. However, in the bladder it is shown that only in 1 out of 10 cases is no dysuria reported while there is DE involvement of the bladder. This translates to a stronger correlation of bladder involvement and dysuria symptoms.

\section{The predictive value of symptoms and endometriosis involvement}

\section{Dyschezia and the ureters}

Unfortunately, endometriosis invasion of the ureters is often asymptomatic or presents with nonspecific symptoms (21), which can lead to silent, obstructive uropathy and renal failure, with a high rate of kidney loss $(23-47 \%)(22)$. 
To detect ureteric involvement, an MRI is the first choice diagnostic tool (21), while it allows proper evaluation of the whole pelvis and helps to decide the surgical approach. In our study we found that dyschezia has a predictive value in detecting ureteric involvement. From an anatomical point of view this can easily be explained, an endometriosis nodule in, or close to the rectum (which causes dyschezia) can also involve or press against the ureter. Translating our research evidence to clinical practice: if endometriosis patients report dyschezia (NRS>6.5), the clinician should be aware of possible ureteric involvement and extend the transvaginal ultrasound with a mandatory MRI (pelvis with kidneys) or at least an ultrasound of the kidneys/ureters.

\section{Dysuria and the bladder}

The majority of patients with bladder endometriosis are symptomatic (dysuria, voiding problems, etc.) (23) (24) (25). This supports our finding of the symptom of dysuria having a predictive value for the presence of bladder endometriosis and is seen clearly in the euler diagram (Figure 1.5). However the finding in the ROC curve was not strong, but we can carefully conclude that even low NRS for dysuria $(>0.5)$, could indicate bladder endometriosis. Translated to clinical practice, patients presenting with very little dysuria symptoms can still have bladder endometriosis.

\section{Importance of classification despite poor clinical correlation}

Despite the limited correlations, anatomical based endometriosis classification remains important. Classification provides a uniform language to communicate, monitor and report the disease (26). This enables standardisation of outcome measures and provides a universal way to communicate about endometriosis in clinical and research settings.

Our study differs from other clinical correlation studies on DE, because we analysed solitary anatomical structures classified with Enzian (compartment A, B and C) related to preoperative pain symptoms as well combinations of compartments $(\mathrm{AB}, \mathrm{AC}, \mathrm{BC}, \mathrm{ABC})$. The results of our study shows that an increase in pain does not correlate with the extent of anatomical involvement. This means that an increase in endometriosis involvement, does not automatically lead to an increase in pain symptoms. This knowledge is new and provides insight in the pain behaviour/symptoms of DE patients. Therefore, clinicians cannot rely on the clinical presentation of DE patients in relation to the disease extent and equally, doctors cannot give information pre-operatively on the severity of the disease.

\section{Strengths and limitations}

An important strength of our study is the fact that this is, to our knowledge, the first study which compared solitary and combinations of anatomical locations in correlation to pain reporting and created ROC curves to determine if certain symptoms could predict the presence of endometriosis in specific compartments. Because of the use of the EQUSUM, data was collected in a uniform way, making it easy to analyse the data without inconsistencies in the datafile.

A limitation of this study is the fact that no medication usage was registered and the NRS pain reporting is pre-operative in patients with and without medication. However, a subgroup analysis within the study from Montanari et al. (5) showed that preoperative use of hormonal therapy did not influence the associations and correlations for reported pain symptoms described by the Enzian. Only a weak correlation was found with the rASRM and dysmenorrhoea when patients used hormonal therapy. So, medication use is obviously of influence, but it seems to have little effect on the results.

A different limitation of this study is that no pathology results are recorded. The endometriosis is staged on the clinical subjective measure of the surgeon and not confirmed by the pathologist. However, there is no debate of a $\mathrm{C} 1$ nodule, whether this is DE of not.

\section{Conclusion}

Our study showed that an increase in pain symptoms does not necessarily lead to an increase in DE involvement. The symptom dyschezia has some predictive value for ureteric involvement, and therefore patients 
with dyschezia should preferably get an MRI of the pelvis or an ultrasound scan of the kidneys/ureters for an optimal surgical workup. Already minor dysuria symptoms can indicate the presence of endometriosis in the bladder. More and more evidence is showing that pain symptoms in deep endometriosis have a multifactorial cause, which partly explains the poor correlations in studies focussing solely on anatomical locations and pain symptoms.

\section{Disclosure of interest}

None declared.

\section{Contribution to authorship}

J.M. performed the analyses together with K.N. and K.B.. J.M, M.B, F.J, A.T, took the lead in writing the manuscript. All authors were responsible for interpreting the data and final review of the manuscript. All authors approved the final manuscript.

\section{Details of ethics approval}

Ethical approval was given by the Medical Ethics Committee of the Leiden University Medical Centre (LUMC) (G20.019).

Funding: The author(s) received no financial support for the research, authorship, and/or publication of this article.

\section{Acknowledgements}

N/A

\section{References}

1. Adamson GD. Endometriosis classification: an update. Curr Opin Obstet Gynecol. 2011;23(4):213-20.

2. Revised American Society for Reproductive Medicine classification of endometriosis: 1996. Fertil Steril. 1997;67(5):817-21.

3. Keckstein J, Ulrich U, Possover M, Schweppe KW. ENZIAN-Klassifikation der tief infiltrierenden Endometriose. Zentralbl Gynakol. 2003;125:291-.

4. Haas D, Oppelt P, Shebl O, Shamiyeh A, Schimetta W, Mayer R. Enzian classification: does it correlate with clinical symptoms and the rASRM score? Acta Obstet Gynecol Scand. 2013;92(5):562-6.

5. Montanari E, Dauser B, Keckstein J, Kirchner E, Nemeth Z, Hudelist G. Association between disease extent and pain symptoms in patients with deep infiltrating endometriosis. Reprod Biomed Online. 2019.

6. Haas D, Shebl O, Shamiyeh A, Oppelt P. The rASRM score and the Enzian classification for endometriosis: their strengths and weaknesses. Acta Obstet Gynecol Scand. 2013;92(1):3-7.

7. Keckstein J, Saridogan E, Ulrich UA, Sillem M, Oppelt P, Schweppe KW, et al. The \#Enzian classification: A comprehensive non-invasive and surgical description system for endometriosis. Acta Obstet Gynecol Scand. 2021;100(7):1165-75.

8. Montanari E, Dauser B, Keckstein J, Kirchner E, Nemeth Z, Hudelist G. Association between disease extent and pain symptoms in patients with deep infiltrating endometriosis. Reprod Biomed Online. 2019;39(5):845-51.

9. Adamson GD, Pasta DJ. Endometriosis fertility index: the new, validated endometriosis staging system. Fertil Steril. 2010;94(5):1609-15.

10. Cornillie FJ, Oosterlynck D, Lauweryns JM, Koninckx PR. Deeply infiltrating pelvic endometriosis: histology and clinical significance. Fertility and Sterility. 1990;53(6):978-83. 
11. Donnez O, Donnez J. Deep endometriosis: The place of laparoscopic shaving. Best Practice \& Research Clinical Obstetrics \& Gynaecology. 2021;71:100-13.

12. Larsson J. eulerr: Area-Proportional Euler and Venn Diagrams with Ellipses. . R package version 610,. 2020;https://cran.r-project.org/package=eulerr.

13. Hosmer Jr DW, Lemeshow S, Sturdivant RX. Applied logistic regression: John Wiley \& Sons; 2013.

14. Pugsley Z, Ballard K. Management of endometriosis in general practice: the pathway to diagnosis. The British journal of general practice : the journal of the Royal College of General Practitioners. 2007;57(539):470-6.

15. Moradi M, Parker M, Sneddon A, Lopez V, Ellwood D. Impact of endometriosis on women's lives: a qualitative study. BMC women's health. 2014;14:123-.

16. Davis-Kankanamge C. The Holistic Approach to Managing Adolescent Endometriosis. Endometriosis in Adolescents: Springer; 2020. p. 679-84.

17. Yong PJ. Deep Dyspareunia in Endometriosis: A Proposed Framework Based on Pain Mechanisms and Genito-Pelvic Pain Penetration Disorder. Sexual Medicine Reviews. 2017;5(4):495-507.

18. Lame IE, Peters ML, Vlaeyen JW, Kleef M, Patijn J. Quality of life in chronic pain is more associated with beliefs about pain, than with pain intensity. Eur J Pain. 2005;9(1):15-24.

19. Lagana AS, La Rosa VL, Rapisarda AMC, Valenti G, Sapia F, Chiofalo B, et al. Anxiety and depression in patients with endometriosis: impact and management challenges. International journal of women's health. 2017;9:323-30.

20. Harte SE, Harris RE, Clauw DJ. The neurobiology of central sensitization. 2018;23(2):e12137.

21. Palla V-V, Karaolanis G, Katafigiotis I, Anastasiou I. Ureteral endometriosis: A systematic literature review. Indian J Urol. 2017;33(4):276-82.

22. Charatsi D, Koukoura O, Ntavela IG, Chintziou F, Gkorila G, Tsagkoulis M, et al. Gastrointestinal and Urinary Tract Endometriosis: A Review on the Commonest Locations of Extrapelvic Endometriosis. Adv Med. 2018;2018:3461209-.

23. Abrao MS, Dias Jr JA, Bellelis P, Podgaec S, Bautzer CR, Gromatsky C. Endometriosis of the ureter and bladder are not associated diseases. Fertility and sterility. 2009;91(5):1662-7.

24. Fauconnier A, Chapron C, Dubuisson J-B, Vieira M, Dousset B, Breart G. Relation between pain symptoms and the anatomic location of deep infiltrating endometriosis. Fertility and sterility. 2002;78(4):719-26.

25. Knabben L, Imboden S, Fellmann B, Nirgianakis K, Kuhn A, Mueller MD. Urinary tract endometriosis in patients with deep infiltrating endometriosis: prevalence, symptoms, management, and proposal for a new clinical classification. Fertility and sterility. 2015;103(1):147-52.

26. Jutel A. Classification, disease, and diagnosis. Perspectives in biology and medicine. 2011;54(2):189-205.

\section{Hosted file}

Tables 31-10-2021 table 1.docx available at https://authorea.com/users/428141/ articles/545290-preoperative-pain-measurements-in-correlation-to-deep-endometriosisclassification-with-enzian

\section{Hosted file}

Tables 31-10-2021 table 2.docx available at https://authorea.com/users/428141/ articles/545290-preoperative-pain-measurements-in-correlation-to-deep-endometriosisclassification-with-enzian 


\section{Hosted file}

Tables 31-10-2021 table 3.docx available at https://authorea.com/users/428141/ articles/545290-preoperative-pain-measurements-in-correlation-to-deep-endometriosisclassification-with-enzian

\section{Hosted file}

Tables 31-10-2021 table 4.docx

available

at

https://authorea.com/users/428141/ articles/545290-preoperative-pain-measurements-in-correlation-to-deep-endometriosisclassification-with-enzian
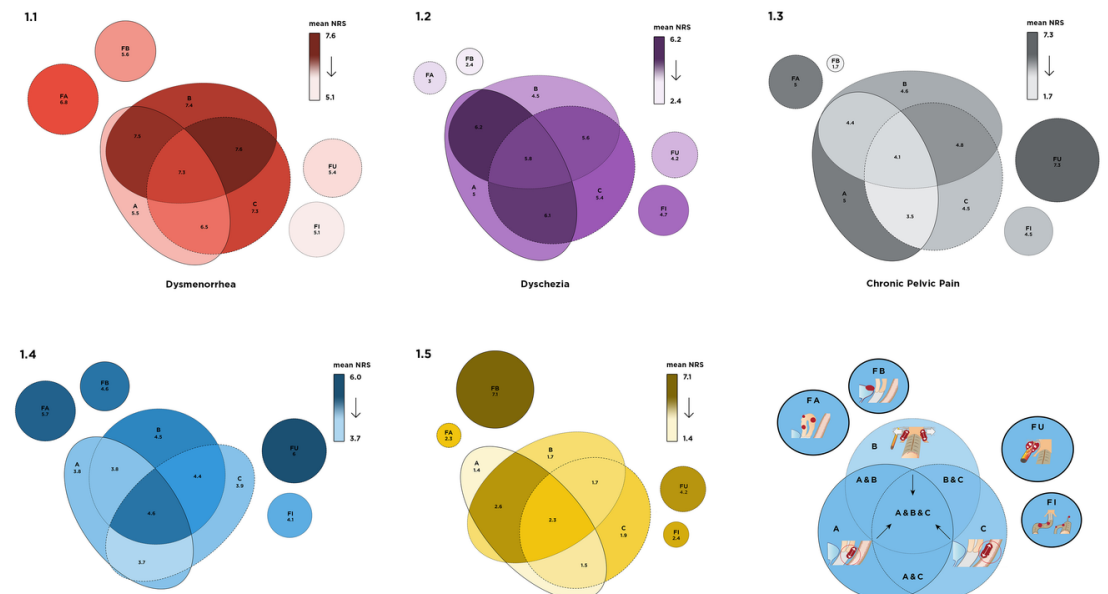

1.5
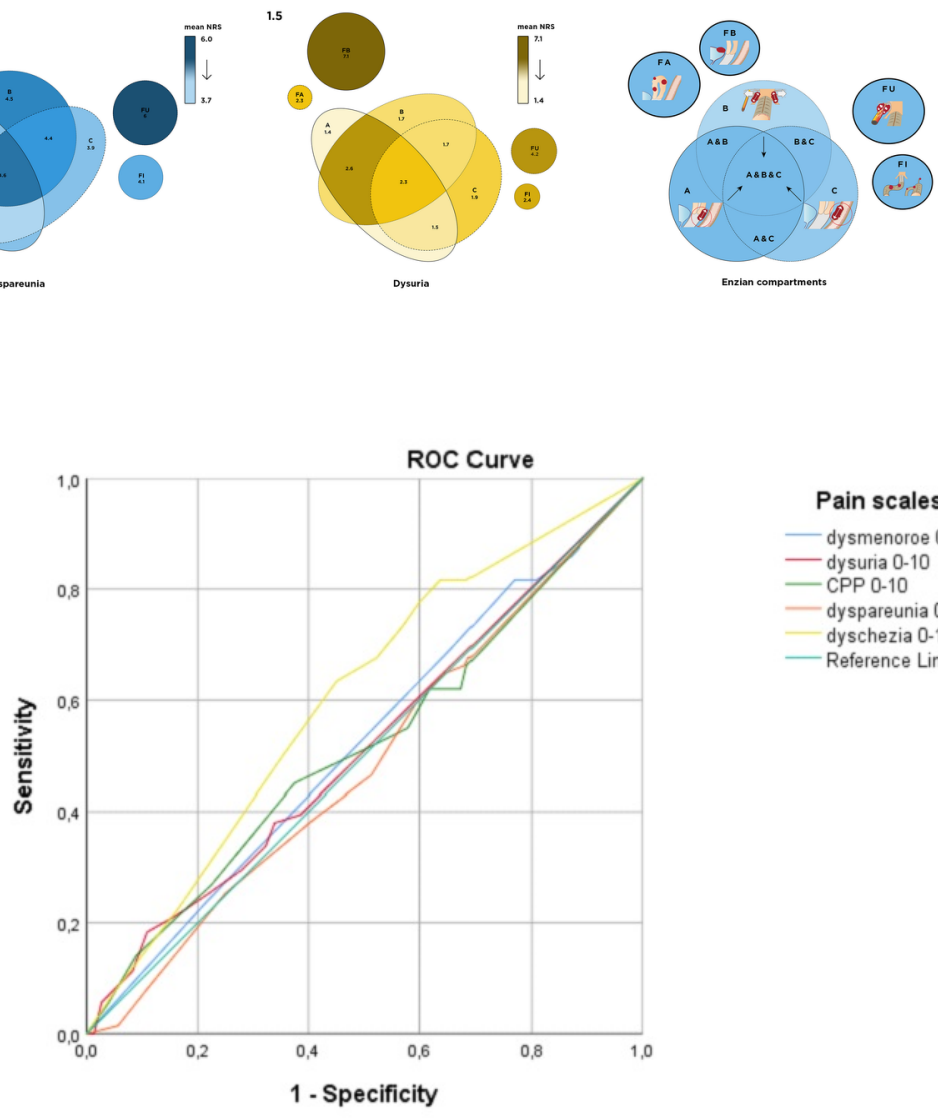

Pain scales

- dysmenoroe 0-10 - dysuria 0-10 - CPP 0-10

- dyspareunia 0-10

dyschezia 0-10

Reference Line

Figure 2.1: ROC curve of Enzian FU (ureter). 


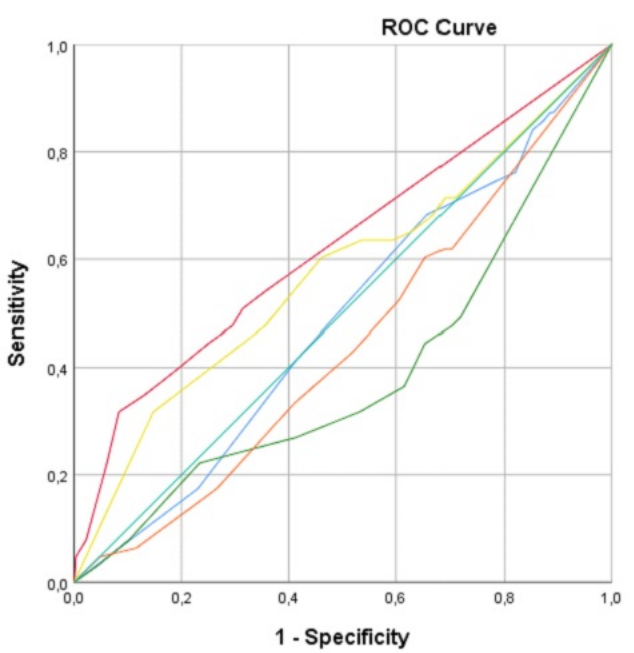

Pain scales

- dysmenoroe 0-10

dysuria 0-10

- dyspareunia 0-10

dyschezia 0-10

- Reference Line

Figure 2.2 ROC curve of Enzian FB (bladder). 